\title{
Determinantes da experimentação do cigarro e do início precoce do tabagismo entre adolescentes escolares em Salvador (BA)*
}

\author{
Determinants of smoking experimentation and initiation \\ among adolescent students in the city of Salvador, Brazil \\ Adelmo de Souza Machado Neto, Tarcisio Matos Andrade, Carlos Napoli, \\ Lilian Carine Souza de Lima Abdon, Mônica Rocha Garcia, Francisco Inácio Bastos
}

\begin{abstract}
Resumo
Objetivo: Identificar os fatores associados à experimentação do cigarro e ao início precoce do tabagismo por adolescentes escolares (11-14 anos). Métodos: Estudo transversal de base populacional com adolescentes escolares (ensino fundamental e médio) de escolas públicas e particulares de Salvador, Bahia. Foi utilizado um protocolo de amostragem em múltiplos estágios. Os participantes da pesquisa responderam a um questionário anônimo, de preenchimento voluntário e autoaplicável. As variáveis dependentes foram o uso experimental do cigarro e o início precoce do tabagismo. Variáveis sociodemográficas, educacionais, relacionadas à influência da mídia e ao consumo de álcool foram consideradas variáveis independentes. Foi realizada a análise de regressão logística multivariada, seguida da análise da adequação do modelo de regressão logística final. Resultados: A prevalência de experimentação do cigarro foi de 16,1\% (1C95\%: 15,1-17,2). Dos 5.347 alunos avaliados, 757 experimentaram cigarros, e $462(61,2 \%)$ desses 757 o fizeram antes dos 15 anos de idade (média de idade de experimentação de cigarros, 13,9 \pm 1,8 anos). 0 início precoce do tabagismo associou-se com o tabagismo paterno $(0 \mathrm{R}=1,53 ; 1 \mathrm{C} 83 \%$ : $1,07-2,19)$ e com a influência da mídia $(\mathrm{OR}=2,43$; 1C83\%: 1,46-4,04). A experimentação de cigarros associou-se com o consumo do álcool ( $\mathrm{OR}=6,04$; 1C83\%: 4,62-7,88), o tabagismo de amigos (OR = 3,18; 1C83\%: 2,54-3,99) e de namorados ( $O R=3,42$; 1C83\%: 1,78-6,56), assim como a intervenção educacional agressiva/coercitiva dos pais $(\mathrm{OR}=1,60 ; 1 \mathrm{C} 83 \%$ : 1,26-2,04). Conclusões: 0 consumo do álcool e a influência de pessoas da convivência do jovem apresentaram uma forte associação com a experimentação de cigarros. 0 tabagismo paterno e a influência da mídia para os produtos do tabaco aumentaram as chances de consumo desta substância.
\end{abstract}

Descritores: Tabagismo; Adolescência; Educação em saúde; Estudos transversais; Fatores epidemiológicos; Brasil.

\begin{abstract}
Objective: To identify the factors associated with smoking experimentation and initiation among adolescent students (11-14 years of age). Methods: A cross-sectional, population-based study involving adolescent (middle and high school) students at public and private schools in the city of Salvador, Brazil. A multiple-stage sampling protocol was used. The participants completed an anonymous, voluntary, self-report questionnaire. The dependent variables were age at smoking experimentation and smoking initiation. Sociodemographic and educational variables, as well as those related to media influence and alcohol consumption, were considered independent variables. Multivariate logistic regression analysis was performed, followed by analysis of the fit of the final logistic regression model. Results: The prevalence of smoking experimentation was $16.1 \%$ (95\% $\mathrm{Cl}: 15.1-17.2)$. Of the 5,347 students evaluated, 757 had experimented with cigarettes, and 462 (61.2\%) of those 757 had done so before the age of 15 (mean age at smoking experimentation, $13.9 \pm 1.8$ years). Smoking initiation was associated with paternal smoking $(\mathrm{OR}=1.53 ; 83 \% \mathrm{Cl}=1.07-2.19)$ and with media influence $(\mathrm{OR}=2.43 ; 83 \% \mathrm{Cl}: 1.46-4.04)$. Smoking experimentation was associated with alcohol consumption ( $\mathrm{OR}=6.04 ; 83 \% \mathrm{Cl}$ : 4.62-7.88), having friends who smoke $(\mathrm{OR}=3.18 ; 83 \% \mathrm{Cl}: 2.54-3.99)$, having a boyfriend/girlfriend who smokes $(\mathrm{OR}=3.42 ; 83 \%$ $\mathrm{Cl}$ : 1.78-6.56), and coercive/aggressive educational interventions by the parents ( $\mathrm{OR}=1.60 ; 83 \% \mathrm{Cl}: 1.26-2.04)$. Conclusions: Alcohol consumption and the influence of peers and relatives who smoke showed a strong association with smoking experimentation. Paternal smoking and media influence were found to increase the chances of adolescent smoking.
\end{abstract}

Keywords: Smoking; Adolescence; Health education; Cross-sectional studies; Epidemiologic factors; Brazil.

* Trabalho realizado no Curso de Pós-Graduação em Medicina e Saúde, Faculdade de Medicina da Bahia, Universidade Federal da Bahia, Salvador (BA) Brasil.

Endereço para correspondência: Adelmo de Souza Machado Neto. Rua Humberto de Campos, 144, sala 1103, Graça, CEP 40150-121, Salvador, BA, Brasil.

Tel 5571 3247-1585. E-mail: machadonetoa@gmail.com

Apoio financeiro: Este estudo recebeu apoio financeiro da Fundação de Amparo à Pesquisa do Estado da Bahia (FAPESB), do Conselho Nacional de Desenvolvimento Científico e Tecnológico (CNPq) e da Secretaria de Ciência, Tecnologia e lnsumos Estratégicos do Ministério da Saúde do Brasil.

Recebido para publicação em 5/7/2010. Aprovado, após revisão, em 4/8/2010.

** A versão completa deste artigo com os anexos está disponível em www.jornaldepneumologia.com.br 


\section{Introdução}

0 tabagismo vem diminuindo na população acima de 18 anos, ${ }^{(1,2)}$ possivelmente devido ao aumento dos investimentos nas campanhas antifumo. ${ }^{(2)}$ Entre os adolescentes, essas campanhas não apresentaram ainda os resultados esperados, e determinantes adicionais do tabagismo estão por ser elucidados. Levantamentos da Organização Mundial da Saúde (OMS) revelaram que, em muitos países, não houve redução desse consumo entre jovens de 13-15 anos, inclusive no Brasil. ${ }^{(3)}$

A prevalência do tabagismo em adolescentes no Sudeste Europeu varia substancialmente $(3,5-42,7 \%) .{ }^{(4)}$ Nos EUA, aproximadamente 50\% dos adolescentes escolares já experimentaram cigarros (uso na vida), com prevalências de 24,9-66,3\%.(5) 0 consumo experimental de cigarros na população geral da América Latina variou de 26,9\% (Brasil) a 66,3\% (Chile). ${ }^{(6)}$ No Brasil, o consumo experimental de cigarros entre os adolescentes apresenta proporções de 5,1\% a $26,9 \% .^{(6-10)}$

Os determinantes para o tabagismo são multifatoriais e estão inter-relacionados. A influência dos pares e pais, ${ }^{(9,11,12)}$ o gênero masculino, ${ }^{(7,9)}$ a prevalência do tabagismo no contexto de vida, ${ }^{(11)}$ os comportamentos antissociais, ${ }^{(11)}$ a reprovação na escola, ${ }^{(12)}$ a baixa autoestima/problemas familiares, ${ }^{(13,14)}$ o estudo em escola privada, ${ }^{(7)}$ a classe socioeconômica menos favorecida, ${ }^{(7,12,15)}$ a influência da mídia ${ }^{(16)}$ e a idade cronológica $>15$ anos estão associados ao início do tabagismo. . $5,7,9,12)^{-}$

0 uso experimental de cigarros por adolescentes escolares constitui um comportamento antissocial e de risco, ${ }^{(7)}$ a exemplo do seu uso subsequente. ${ }^{(17)}$ Identificar determinantes da iniciação do tabagismo pode subsidiar políticas públicas adequadas. Este estudo teve como objetivo identificar os fatores associados à experimentação de cigarros e ao início precoce do tabagismo entre adolescentes escolares da cidade do Salvador (BA).

\section{Métodos}

Foi realizado um inquérito transversal. Utilizaram-se dados relativos ao uso experimental de cigarros provenientes de um levantamento sobre o consumo de substâncias psicoativas entre adolescentes escolares, realizado na cidade de Salvador em 2008, englobando 47 escolas públicas e particulares, em 15 regiões administrativas.

0 instrumento para a coleta de dados foi um formulário para leitura óptica, anônimo e autoaplicável, contendo 93 questões sobre o uso de substâncias psicoativas e comportamentos relacionados a essas práticas, com base em questionários anteriores. ${ }^{(18)} 0$ questionário se mostrou adequado em um estudo de confiabilidade. $^{(18)}$

As variáveis compreenderam as relações dos adolescentes com a escola e com os pais; opiniões sobre os meios de comunicação e campanhas contra o uso de tabaco, álcool e maconha; consumo de substâncias psicoativas na vida, no ano e no mês anterior à aplicação do questionário; padrões de consumo dessas substâncias; critérios de dependência da nicotina (segundo os critérios de Fagerström), do álcool e da maconha (segundo os critérios da OMS); e critérios definidores de abuso de álcool e maconha. ${ }^{(18)}$

Adolescentes escolares matriculados no ensino fundamental ( $5^{\mathrm{a}-} 8^{\mathrm{a}}$ séries) e médio $\left(1^{\circ}-3^{\circ}\right.$ anos) de escolas públicas e particulares na cidade de Salvador (BA), com idades entre 11 e 19 anos, de ambos os sexos, compuseram a população estudada. Segundo o Censo Educacional de Salvador de 2000, o número de alunos matriculados (ensino fundamental e médio) era de 715.877. Desses, 538.364 cursavam o ensino fundamental (467.961 em escolas públicas estaduais e municipais e 70.403 em escolas particulares) e 177.513 cursavam o ensino médio (156.784 na rede federal e estadual e 20.729 na rede privada). A cidade de Salvador conta com 740 estabelecimentos escolares públicos e privados que lecionam da $5^{\text {a }}$ série do ensino fundamental ao $3^{\circ}$ ano do ensino médio. ${ }^{(18)}$

A amostragem compreendeu uma primeira etapa aleatória, estratificada por conglomerados, sorteando-se 77 escolas. Foram realizados sorteios independentes dos estabelecimentos de ensino, respeitando-se o critério de inclusão de $10 \%$ das escolas do ensino fundamental e de $10 \%$ das escolas do ensino médio do total de escolas. As escolas com mais de 150 matrículas foram consideradas elegíveis para o estudo. Dentre as escolas sorteadas, 47 aceitaram participar do estudo (29 estaduais, 4 municipais, 2 federais e 12 particulares). Cada escola 
foi, então, estratificada de acordo com a sua natureza (pública ou privada) e nível de ensino (fundamental ou médio). Sortearam-se, então, as turmas. Todos os alunos de cada turma sorteada foram convidados a participar do estudo.

Foram aplicados 6.500 questionários, $3.801(58,5 \%)$ deles no turno da manhã e $2.699(41,5 \%)$ no turno da tarde. A composição da amostra refletiu a proporção de alunos em Salvador, segundo a natureza da escola (pública ou privada) e o nível do ensino (fundamental ou médio). Dos alunos que participaram da amostra, $82,2 \%$ eram da rede pública e $54,1 \%$, do ensino fundamental.
0 número mínimo necessário para estimar a proporção do consumo de uma das substâncias envolvidas no estudo levou em conta os seguintes fatores: a população de escolares da $5^{a}$ série do ensino fundamental ao $3^{\circ}$ ano do ensino médio, a precisão do estudo $(20,0 \%)$, a prevalência esperada de consumo, ${ }^{(7)}$ o efeito do desenho ${ }^{(1,5)}$ e o nível de confiança (95\%). Considerou-se, para o efeito de cálculo, a droga de maior consumo (álcool), perfazendo um total de 6.267 alunos: 1.624 do ensino fundamental e 4.643 do ensino médio. Considerou-se uma perda de 50\%, potencialmente associada a lacunas de informação. Essa foi uma estratégia

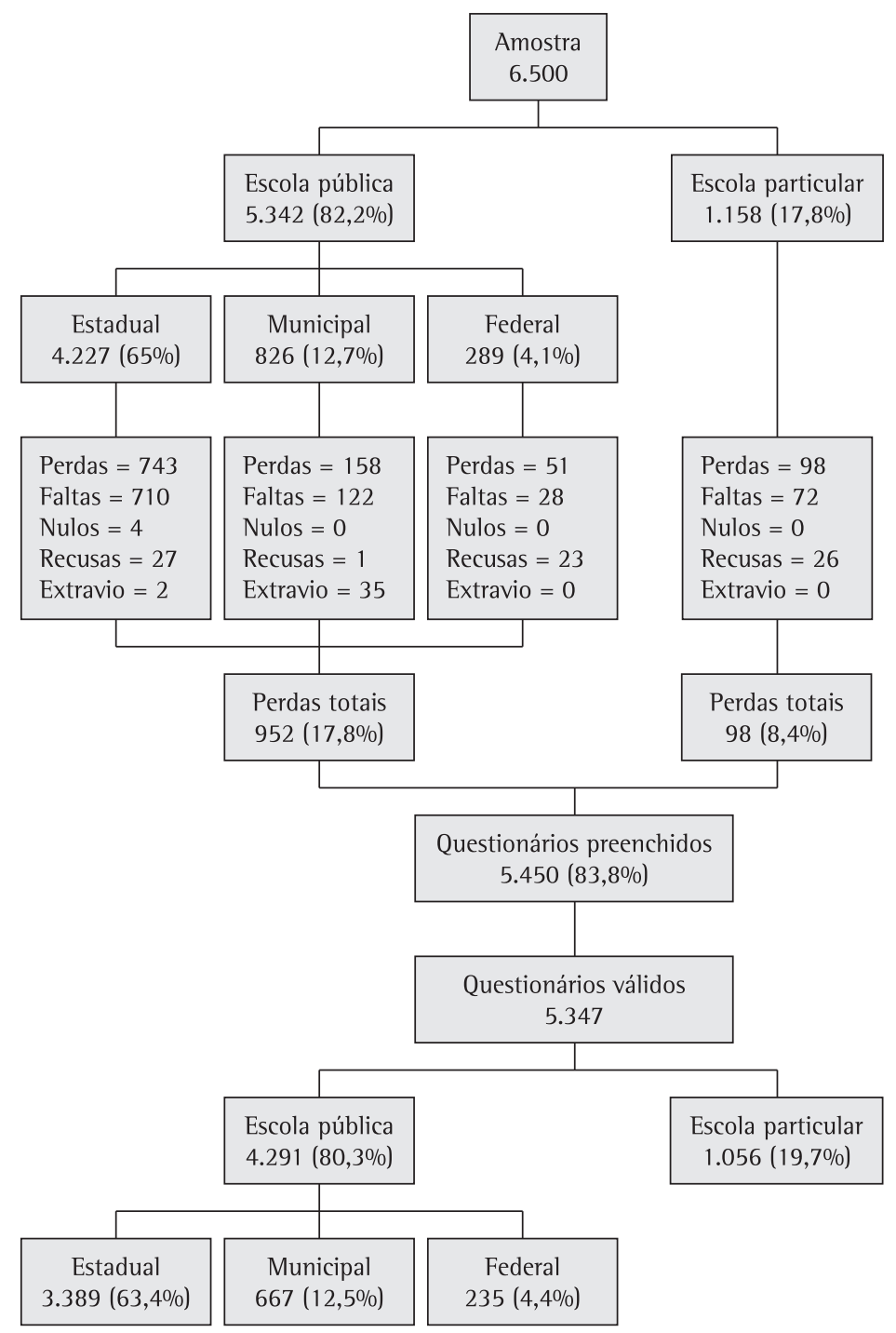

Figura 1 - Representação esquemática da amostra obtida no levantamento sobre o consumo de substâncias psicoativas por adolescentes escolares da cidade de Salvador (BA), 2008. 
Tabela 1 - ldade de experimentação de cigarros entre adolescentes escolares da cidade de Salvador, 2008.

\begin{tabular}{ccc}
\hline ldade, anos & $\mathrm{n}(\%)$ & $1 \mathrm{C} 95 \%$ \\
\hline 11 & $89(11,8)$ & $9,7-14,3$ \\
$12-14$ & $373(49,4)$ & $45,9-52,9$ \\
$15-18$ & $293(38,8)$ & $35,4-42,3$ \\
Total & $755(100)$ & \\
\hline
\end{tabular}

que preservou o poder do estudo em relação ao cálculo quanto à relação entre indivíduos expostos e não expostos na comparação de variáveis. A análise do início precoce do tabagismo no presente estudo compreendeu 783 alunos, que responderam ter experimentado cigarros.

0 instrumento de pesquisa foi aplicado nas escolas por estudantes de medicina e agentes de saúde da Aliança de Redução de Danos Fátima Cavalcanti da Universidade Federal da Bahia (UFBA), que foram previamente treinados. Os questionários foram aplicados durante os horários de aulas das turmas incluídas no estudo, entre os dias 7 de abril e 13 de junho de 2008.

Aplicou-se a técnica denominada bogus pipeline, ${ }^{(19)}$ técnica essa de estimulação para responder à pesquisa, com o objetivo de minimizar erros sistemáticos e informações inconsistentes. A pesquisa foi aprovada pelo Comitê de Ética em Pesquisa da UFBA. Precedendo a aplicação do questionário, o projeto foi apresentado aos diretores das escolas selecionadas, e o diretor de cada escola foi convidado a assinar um termo de consentimento informado, assim como a definir a data de aplicação do instrumento. De acordo com as orientações do comitê de ética, não foi necessário obter o consentimento informado de cada aluno individualmente, dado o caráter anônimo do instrumento.
Os alunos foram assegurados de que os dados eram confidenciais e que eles tinham a opção de participar ou não do estudo, ou de não responder às perguntas que considerassem impróprias.

0 uso experimental de cigarros (na vida) e o início precoce do tabagismo (11-14 anos) foram definidos como variáveis dependentes, e as variáveis independentes incluíram variáveis sociodemográficas, aquelas relacionadas à exposição ao tabagismo de terceiros, aquelas relacionadas aos aspectos educacionais e à mídia, assim como ao consumo de álcool no ano/mês (Anexo 1, disponível na versão eletrônica desta publicação). ${ }^{(18)}$

Os critérios de inclusão compreenderam o recebimento de questionários em bom estado (sem rasgos/rasuras que impedissem a leitura), respondidos em sala de aula, por alunos da $5^{a}$ série do ensino fundamental ao $3^{\circ}$ ano do ensino médio, com idades entre 11 e 19 anos. Foram consideradas as respostas em sintonia com o enunciado das questões, sendo válidas para a análise da frequência de consumo e de dependência de determinada substância psicoativa as respostas as quais o aluno respondeu ter consumido aquela substância pelo menos uma vez na vida, além de responder as questões a ela correspondentes.

Os dados foram transcritos para um banco de dados (6.500 registros) por leitura óptica. A análise dos dados foi realizada com os programas Statistical Package for the Social Sciences, versão 12.0 (SPSS Inc., Chicago, IL, EUA) e PEPI for Windows versão 4.1 (Sagebush Press, Salt Lake City, UT, EUA). As variáveis descritivas são apresentadas em frequência, média e valores mínimos e máximos.

Tabela 2 - Resultados do modelo final da análise de regressão logística multivariada a para a idade de uso experimental de cigarros por escolares (11-14 anos) na cidade de Salvador (BA), 2008.

\begin{tabular}{lccccc}
\hline \multicolumn{1}{c}{ Variável independente } & $\beta$ & EP $\beta$ & OR & 1C83\% & $p$ \\
\hline Gênero masculino & $-0,607$ & 0,209 & 0,54 & $0,41-0,73$ & 0,004 \\
Cor da pele branca/asiática & $-0,309$ & 0,223 & 0,73 & $0,54-1,00$ & 0,167 \\
Classe econômica B1-A2 & $-0,466$ & 0,231 & 0,63 & $0,46-0,86$ & 0,044 \\
Tabagismo paterno & 0,423 & 0,263 & 1,53 & $1,07-2,19$ & 0,107 \\
Mídia dos produtos do tabaco & 0,889 & 0,370 & 2,43 & $1,46-4,04$ & 0,016 \\
Constante & 0,863 & 0,186 & 2,37 & & 0,000 \\
\hline
\end{tabular}

avariáveis incluídas no modelo inicial: dependência administrativa da escola; gênero; cor da pele; classe econômica; tabagismo paterno ou materno; amigos que fumam; professor que fuma; mídia dos produtos do tabaco; consumo de álcool no ano ou mês; abordagem dos professores sobre consumo de drogas; e frequência com que os pais falam sobre drogas. 
Determinou-se o 1C95\% para a precisão das prevalências. $\mathrm{Na}$ análise de regressão logística, ${ }^{(20)}$ foram utilizados dois critérios para a seleção das variáveis independentes a serem incluídas no modelo: variáveis que apresentaram associações estatisticamente significantes $(p \leq 0,25)$ na análise univariada e variáveis que apresentaram associações relevantes com os desfechos na literatura consultada.

As variáveis foram mantidas no modelo a partir da consulta à literatura, levando-se em consideração sua possível atuação como um preditor associado à variável dependente e seu valor de $p$ nos modelos parciais e final. Para a manutenção das variáveis no modelo final, foram considerados $p \leq 0,17$ e 1C83\%. Procedeu-se à análise da adequação do modelo logístico final mediante o teste estatístico de Hosmer-Lemeshow e a análise dos resíduos (unstandardized, standardized, studentized, deviance, levarage, distâncias de Cook e DFBETAs).

\section{Resultados}

Dos 6.500 questionários distribuídos, 5.450 $(83,8 \%)$ foram preenchidos. As perdas se deveram, na maioria dos casos, a informações ausentes $(14,4 \%)$, compreendendo faltas às aulas no dia da aplicação do questionário (14,3\%) e questionários nulos $(0,1 \%)$. Houve também a perda propriamente dita do questionário $(1,8 \%)$, assim como aquela constituída por recusas $(1,2 \%)$ e extravios $(0,6 \%)$. 0 total de questionários válidos foi de 5.347 (Figura 1).

A média de idade dos jovens da amostra ( $\mathrm{n}=4.883$ ) foi de 15,0 $\pm 1,8$ anos (1C95\%: 15,0-15,1 anos). 0 tabagismo na vida foi de 16,1\% (783/4.855; 1C95\%: 15,1-17,2). A média de idade do uso experimental do cigarro $(n=757)$ foi de 13,9 $\pm 1,8$ anos (1C95\%: 13,8-14,3 anos). A experimentação do cigarro ocorreu precocemente (11 anos de idade) em 11,8\% da amostra, e mais da metade dos jovens (462/755; 61,2\%; 1C95\%: 57,7-65,6\%) experimentou cigarros antes dos 15 anos (Tabela 1).

No modelo ajustado, as variáveis que se mostraram independentemente associadas ao início precoce do tabagismo foram as seguintes: ser do gênero masculino, autodenominar sua cor da pele como branca/asiática e pertencer à classe econômica B1-A2 (valores de beta negativos). 0 tabagismo paterno e a influência da mídia dos produtos de tabaco se mostraram associadas a maiores ORs (Tabela 2). 0 teste de qualidade do ajuste do modelo logístico final apresentou um valor de qui-quadrado de 4,291, com 7 graus de liberdade e $p=0,746$ para o teste estatístico de Hosmer-Lemeshow. A análise dos resíduos revelou um ajuste adequado.

Dentre os motivos assinalados pelos adolescentes da amostra para o consumo experimental de cigarros, a curiosidade foi o mais frequentemente mencionado, com uma proporção menor de respostas que mencionavam "nenhuma influência". O efeito da nicotina (prazer e relaxamento) e a influência de amigos constituíram motivos adicionais frequentemente assinalados (Tabela 3).

$\mathrm{Na}$ análise multivariada, os alunos das escolas particulares, aqueles que cursavam o ensino médio e os que se autodenominaram com cor da pele branca/asiática apresentaram valores de beta negativos. No modelo final, ter idade entre 15 e 19 anos, ser filho de pais separados, haver fumantes entre pessoas da convivência do jovem (pai, mãe, irmãos, amigos e namorados), ter pais que raramente ou nunca conversam sobre drogas,

Tabela 3 - Motivações para o uso experimental do cigarro relatadas por adolescentes escolares na cidade de Salvador (BA), 2008.

\begin{tabular}{|c|c|c|c|}
\hline \multirow[t]{2}{*}{ Variáveis } & \multicolumn{3}{|c|}{$\begin{array}{c}\text { Uso experimental } \\
\text { de cigarros }\end{array}$} \\
\hline & $n$ & $\%$ & 1C95\% \\
\hline Curiosidade & 460 & 60,4 & $56,9-63,8$ \\
\hline Influência de amigos & 134 & 17,6 & $15-20,4$ \\
\hline Prazer & 105 & 13,8 & $11,5-16,4$ \\
\hline Relaxamento & 70 & 9,2 & $7,3-11,4$ \\
\hline Poder & 35 & 4,6 & $3,3-6,3$ \\
\hline Redução da ansiedade & 25 & 3,3 & $2,2-4,8$ \\
\hline Desinibição & 19 & 2,5 & $1,6-3,9$ \\
\hline Influência da televisão & 14 & 1,8 & $1,1-3,1$ \\
\hline Influência do pai & 13 & 1,7 & $1-2,9$ \\
\hline $\begin{array}{l}\text { Pressão do grupo de } \\
\text { relacionamento }\end{array}$ & 13 & 1,7 & $1-2,9$ \\
\hline Autoafirmação & 12 & 1,6 & $1-2,7$ \\
\hline $\begin{array}{l}\text { Dificuldade de } \\
\text { relacionamento social }\end{array}$ & 10 & 1,3 & $0,7-2,7$ \\
\hline Influência da mídia ${ }^{a}$ & 8 & 1,0 & $0,5-2,1$ \\
\hline Influência dos professores & 6 & 0,8 & $0,4-1,7$ \\
\hline Influência da mãe & 4 & 0,5 & $0,2-1,3$ \\
\hline Nenhuma influência & 116 & 15,2 & $12,8-17,9$ \\
\hline Outras influências & 18 & 2,4 & $1,5-3,7$ \\
\hline
\end{tabular}

${ }^{a}$ Mídia indireta: filmes, espetáculos, etc. 
ser influenciado pela mídia e ser corrigido pelos pais de forma agressiva/coercitiva se mostraram associados à iniciação do consumo (Tabela 4). 0 consumo de álcool apresentou forte associação com o uso experimental de cigarros $(\mathrm{OR}=6,04$; $1 \mathrm{C} 83 \%=4,62-7,88)$.

0 teste de qualidade do ajuste do modelo logístico apresentou um valor de qui-quadrado de 7,516, com 8 graus de liberdade e $p=0,482$. $A$ análise dos resíduos evidenciou um ajuste adequado.

\section{Discussão}

Os resultados do presente estudo são reprodutíveis, considerando-se que o questionário utilizado apresentou um grau de confiabilidade aceitável. ${ }^{(18)}$ A seleção de escolas e de alunos foi aleatória, as perdas por recusa dos alunos foram inferiores a 5\%, e a amostra das escolas e dos alunos foi representativa para a cidade de Salvador (compreendendo escolas públicas e particulares de 15 regiões administrativas).

A leitura óptica reduziu a níveis despreziveis possíveis vícios de informação relacionados à transcrição, e o tamanho final da amostra correspondeu a um poder de estudo de 97\%.

A utilização da regressão logística com o método de Hosmer-Lemeshow goodness of fit é um dos aspectos metodológicos relevantes. Hosmer et al. observaram que, entre 569 artigos publicados no American Journal of Public Health, $20 \%$ dos autores utilizaram a regressão logística, mas apenas 5\% deles realizaram a análise da adequação do modelo logístico final. ${ }^{(20)}$

0 estudo procurou minimizar suas limitações. 0 viés de informação (erro sistemático) no preenchimento do questionário, inerente ao próprio método, foi minimizado pelo estudo da confiabilidade, ${ }^{(18)}$ com a garantia de confidencialidade e anonimato associados à técnica denominada bogus pipeline. ${ }^{(19)} 0$ papel de variáveis de confusão ou daquelas modificadoras de efeito foi minimizado com a aplicação da regressão logística. Os resultados do estudo são aplicáveis aos alunos matriculados nos turnos matutino/vespertino, não se aplicando àqueles no turno noturno e aos jovens que estão fora da escola.

A prevalência do consumo experimental de cigarros no presente estudo foi menor do que aquelas observadas na União Europeia, onde a proporção de escolares de 17-18 anos que havia fumado pelo menos uma vez na vida variou entre $72 \%$ e $82 \% .{ }^{(21)}$ Nos EUA, apesar da tendência de redução do tabagismo, ${ }^{(2)}$ a prevalência de tabagismo na vida $(50,3 \%)$ foi superior à encontrada no presente estudo. ${ }^{(5)} \mathrm{A}$ metodologia

Tabela 4 - Resultados do modelo final da análise de regressão logística multivariadaa para o uso experimental de cigarros por escolares na cidade de Salvador (BA), 2008.

\begin{tabular}{lrcccc}
\hline \multicolumn{1}{c}{ Variáveis independentes } & $\beta$ & EP $\beta$ & OR & IC83\% & p \\
\hline Dependência administrativa particular & $-0,377$ & 0,179 & 0,69 & $0,54-0,88$ & 0,035 \\
Ensino médio & $-0,409$ & 0,223 & 0,66 & $0,49-0,90$ & 0,067 \\
Cor da pele branca/asiática & $-0,397$ & 0,166 & 0,67 & $0,54-0,84$ & 0,017 \\
ldade 15-19 anos & 1,227 & 0,262 & 3,41 & $2,38-4,88$ & 0,000 \\
Estado civil dos pais (vivem separadamente) & 0,360 & 0,152 & 1,43 & $1,16-1,77$ & 0,018 \\
Tabagismo paterno & 0,781 & 0,200 & 2,18 & $1,66-2,87$ & 0,000 \\
Tabagismo materno & 0,752 & 0,237 & 2,12 & $1,53-2,93$ & 0,002 \\
Tabagismo de irmãos & 0,488 & 0,244 & 1,63 & $1,17-2,28$ & 0,045 \\
Tabagismo de amigos & 1,157 & 0,165 & 3,18 & $2,54-3,99$ & 0,000 \\
Tabagismo de namorados & 1,230 & 0,475 & 3,42 & $1,78-6,56$ & 0,010 \\
Pais raramente ou nunca falam sobre drogas & 0,353 & 0,153 & 1,42 & $1,15-1,76$ & 0,021 \\
Mídia para promoção do tabagismo & 0,691 & 0,261 & 1,99 & $1,39-2,86$ & 0,008 \\
Consumo de álcool no mês ou ano & 1,798 & 0,194 & 6,04 & $4,62-7,88$ & 0,000 \\
Correção dos pais de forma agressiva/coercitiva & 0,472 & 0,177 & 1,60 & $1,26-2,04$ & 0,008 \\
Constante & $-3,961$ & 0,272 & 0,02 & & 0,000 \\
\hline
\end{tabular}

aVariáveis incluídas no modelo inicial: dependência administrativa da escola; gênero; cor da pele; religião; classe econômica; estado civil dos pais; com quem vive; tabagismo paterno ou materno; tabagismo de familiares (irmãos, primos, tios); tabagismo dos pares (amigos, namorados); tabagismo do professor; abordagem dos professores sobre consumo de drogas; frequência com que os pais abordam o tema droga com seus filhos; mídia dos produtos do tabaco; pais proibiriam o uso de cigarros; correção com diálogo/limites; correção de forma agressiva/coercitiva; consumo de álcool no ano ou mês. 
do estudo, os aspectos socioculturais e o momento histórico de cada localidade podem influenciar a prevalência do tabagismo, ${ }^{(2,3,5,5,6,21)} 0$ que explicaria as diferenças observadas.

0 Brasil é o país da América Latina que apresenta a mais baixa prevalência de experimentação de cigarros, com uma prevalência de consumo de cigarros na vida correspondente a aproximadamente metade daquela observada nos demais países latino-americanos. Nesses países, a prevalência de consumo de cigarros na vida variou de 26,9\% (no Brasil) a 66,3\% (no Chile), com uma prevalência média de $42,4 \%{ }^{(6)}$ Os dados obtidos nesta pesquisa se assemelham aos de um estudo realizado com escolares do ensino fundamental e médio de Salvador, no qual $17,7 \%$ dos escolares haviam fumado na vida. (10) Os resultados observados na pesquisa atual foram inferiores àqueles de um levantamento anterior entre escolares de Salvador $(46 \%)^{(9)}$ e de um estudo conduzido pelo Instituto Nacional do Câncer $(29,3 \%){ }^{(22)}$ Esse achado pode estar associado a uma tendência de redução do tabagismo em adolescentes.

Em 2006, durante a fase piloto da pesquisa, observou-se que $12,6 \%$ dos jovens fizeram uso experimental de cigarros. Apesar de ser um estudo piloto, o tamanho da amostra correspondeu a um poder maior do que $90 \%$ para a estimativa da prevalência do tabagismo. ${ }^{(18)}$ A idade de uso experimental de cigarros observada no estudo (11-14 anos) é corroborada por outros levantamentos. ${ }^{(9,16,18)} 0$ gênero masculino, a cor da pele branca/asiática e a classe econômica B1-A2 reduziram as ORs para o início precoce do tabagismo. Esses resultados são discordantes de dados de levantamentos anteriores, nos quais uma maior prevalência desse consumo entre adolescentes de cor branca ${ }^{(5)}$ e do gênero masculino foi observada. ${ }^{(3,5)}$ Pertencer a uma classe econômica menos privilegiada pode determinar o consumo dessa substância. . $^{(1,5)}$

0 tabagismo paterno e a mídia indireta que promove o tabagismo (filmes e espetáculos) aumentam as chances para o uso experimental de cigarros e o início precoce do tabagismo. lsso pode ser explicado pelo comportamento inadequado dos pais, que é copiado pelos filhos, ${ }^{(11,12)}$ e a falta de desenvolvimento de habilidades sociais nessa faixa etária. Não foram encontrados estudos que abordassem diretamente o início precoce do tabagismo.
A curiosidade, o prazer, o relaxamento e a influência de amigos foram as motivações mais frequentes para o tabagismo, o que está em harmonia com a literatura. ${ }^{(11,12,16,23)}$ Uma proporção importante de alunos não percebia, entretanto, qualquer influência. Habitualmente, há um conjunto de fatores que contribuem para o consumo de uma substância psicoativa e a sua inter-relação pode dificultar a individualização de seu papel relativo..$^{(6,11,13,24)}$

$A$ análise ajustada permite inferir que estudar em uma instituição privada ${ }^{(12)}$ e estar no curso médio reduziram as chances de experimentação de cigarros, corroborando achados de um estudo anterior no Brasil, ${ }^{(15)}$ ainda que em contraposição a outros estudos. ${ }^{(5,7,12)}$ Levantamentos realizados nos EUA e na América Latina não evidenciaram uma associação do tabagismo na vida com a natureza administrativa da escola. ${ }^{(5,6,6)}$ A discordância ou a concordância dos dados da atual pesquisa com os de outros levantamentos parece estar associada às características da amostra e ao momento da coleta dos dados de um estudo transversal. As características socioculturais de cada população e seus momentos históricos podem influenciar os achados.

Ter pais que vivem separados (definidos, neste estudo, como divorciados, desquitados, solteiros ou viúvos) pode aumentar a OR para o início precoce do tabagismo, ${ }^{(12,25)}$ possivelmente, na vigência de conflitos familiares. ${ }^{(13)}$ No presente estudo, observou-se a associação (aumento da OR) do início precoce do tabagismo com o tabagismo dos pais, irmãos e, principalmente, dos pares (amigos e namorados), como descrito em estudos anteriores. . $^{(9,11,12,26-28)}$

A raridade/ausência de esclarecimento sobre drogas aos filhos pelos pais e a não proibição do consumo de cigarros aumentaram as chances do seu uso experimental, ${ }^{(13,14,16,29)}$ uma vez que a falta de diálogo e a ausência de limites podem aumentar os riscos de comportamentos antissociais, incluindo o consumo do cigarro e o uso de outras substâncias. ${ }^{(11,23)} 0$ presente estudo não corrobora dados de levantamentos anteriores quanto à variável "proibição dos pais para o consumo do cigarro por seus filhos", a qual não foi selecionada para o modelo final.

Pais que raramente ou nunca falam sobre drogas e que têm uma forma de correção agressiva/coercitiva se mostraram associados a maiores chances de que seus filhos experimentem 
o cigarro. A atitude adequada da família pode constituir um fator de proteção frente a comportamentos antissociais e ao consumo de substâncias psicoativas, ${ }^{(11,14)}$ contribuindo para que o jovem desenvolva habilidades sociais. ${ }^{(30)}$ Um estudo revelou que, ainda que de forma indireta, a promoção do tabagismo pela mídia influencia o consumo de cigarros, ${ }^{(16)}$ o que foi observado na atual pesquisa.

No presente estudo, o consumo de bebidas alcoólicas pelo adolescente no ano ou no mês anterior à aplicação do questionário representou um fator de risco importante para o uso experimental de cigarros. Alguns estudos têm revelado essa associação em relação a outras substâncias psicoativas, ${ }^{(6,28)}$ mas não há relatos na literatura no que diz respeito ao uso experimental de cigarros. Fundamentando-se nesse achado, pode-se inferir que o sucesso na redução do tabagismo entre os jovens deve estar integrado a políticas públicas de prevenção e atenção ao consumo de álcool nessa população. 0 tabagismo e o consumo de álcool e de drogas ilícitas constituem comportamentos de risco inter-relacionados..$^{(5,7)}$

Estudos de coorte seriam talvez adequados para confirmar os presentes achados; porém, para tanto, o adolescente teria de ser identificado, o que poderia comprometer a confiabilidade das informações. As recusas de algumas escolas particulares em participar da pesquisa reforçam essa dificuldade. Entretanto, há evidências de que questionários anônimos $\mathrm{e}$ autoaplicáveis aumentam as possibilidades de respostas positivas quanto ao tabagismo. ${ }^{(19)}$ Há na literatura poucos estudos de coorte nesse campo específico. ${ }^{(11,23)}$

A despeito das limitações de um estudo transversal, os achados revelam que uma proporção importante dos jovens da amostra referiu ter experimentado cigarros. 0 tabagismo dos pares e familiares se mostrou associado ao uso experimental de cigarros, e o tabagismo paterno aumentou as chances do início precoce do tabagismo.

Os jovens com idades entre 15 e 19 anos apresentaram maiores chances de uso experimental de cigarros. A curiosidade, o efeito da substância (prazer e relaxamento) e a influência dos amigos constituíram as principais motivações identificadas para o início precoce do tabagismo. A mídia, a falta de esclarecimento dos pais quanto a essa substância para seus filhos e a correção dos pais de forma agressiva/ coercitiva também se mostraram determinantes para o início precoce do tabagismo. Além disso, observou-se uma forte associação do consumo de álcool com o uso experimental do cigarro, sendo esse aspecto um diferencial do presente estudo.

\section{Agradecimentos}

Agradecemosà Dra. Ana Paula Souza Machado e à Dra. Ana Carmen Costa Dias; aos acadêmicos de medicina Ana Carolina Rocha Garcia, Lauro Reis Santana e Cyntia Sampaio, que colaboram na coleta dos dados para a pesquisa; à Gisele Ghiraldi, que contribuiu durante todo o projeto desde a sua concepção e que coordenou todo o grupo de pesquisa durante a coleta, também participando da análise dos dados; e a Annibal Sylvane Neto, Professor do Departamento de Medicina Preventiva da Faculdade de Medicina da Bahia da UFBA, que nos apoiou durante o projeto de pesquisa.

Os anexos deste artigo estão disponível na versão on-line do Jornal Brasileiro de Pneumologia: www.jornaldepneumologia.com.br

\section{Referências}

1. Monteiro CA, Cavalcante TM, Moura EC, Claro RM, Szwarcwald CL. Population-based evidence of a strong decline in the prevalence of smokers in Brazil (19892003). Bull World Health Organ. 2007;85(7):527-34.

2. Farrelly MC, Pechacek TF, Thomas KY, Nelson D. The impact of tobacco control programs on adult smoking. Am J Public Health. 2008;98(2):304-9.

3. Warren $\mathrm{CW}$, Lea $\mathrm{V}$, Lee J, Jones NR, Asma S, McKenna M. Change in tobacco use among 13-15 year olds between 1999 and 2008: findings from the Global Youth Tobacco Survey. Glob Health Promot. 2009;16(2 Suppl):38-90.

4. World Health Organization; South-East Europe Health network. Reversing the tobacco epidemic. Saving lives in southeastern Europe. Copenhagen: WHO Regional Office for Europe; 2008.

5. Eaton DK, Kann L, Kinchen S, Shanklin S, Ross J, Hawkins J, et al. Youth risk behavior surveillance--United States, 2007. MMWR Surveill Summ. 2008;57(4):1-131.

6. Comisión Interamericana para el Control del Abuso de Drogas (CICAD). Jóvenes y drogas en países sudamericanos: un desafío para las políticas públicas: primer estudio comparativo sobre uso de drogas en población escolar secundaria de Argentina, Bolivia, Brasil, Colombia, Chile, Ecuador, Paraguay, Perú y Uruguay. Lima: Tetis Graf E.I.R.L.; 2006.

7. Carlini-Cotrin B, Gazal-Carvalho C, Gouveia N. Comportamentos de saúde entre jovens estudantes das redes pública e privada da área metropolitana do Estado de São Paulo. Rev Saude Publica 2000;34(6):636-45. 
8. Carlini EA, Galduróz JC, Noto AR, Nappo SA. I Levantamento Domiciliar sobre o Uso de Drogas Psicotrópicas no Brasil: Estudo Envolvendo as 107 Maiores Cidades do País - 2001. São Paulo: Centro Brasileiro de Informações sobre Drogas Psicotrópicas, Universidade Federal de São Paulo; 2002.

9. Machado Neto AS, Cruz AA. Smoking among school adolescents in Salvador, Brazil. J Bras Pneumol. 2004;29(5):264-72.

10. Galduroz JC, Noto AR, Fonseca AM, Carlini EA. V Levantamento nacional sobre o consumo de drogas psicotrópicas entre estudantes do ensino fundamental e médio da rede pública de ensino nas 27 capitais brasileiras-2004. São Paulo: Centro Brasileiro de Informações sobre Drogas Psicotrópicas, Universidade Federal de São Paulo; 2005.

11. Graña JL. Análise dos fatores de risco e de proteção para o consumo de drogas em adolescentes. In: Marinho ML, Caballo VE, editors. Psicologia clínica e da saúde. Londrina: UEL; 2001. p. 55-75.

12. Silva MP, Silva RM, Botelho C. Factors associated with cigarette experimentation among adolescents. J Bras Pneumol. 2008;34(11):927-35.

13. Chávez LM, Andrade D. La escuela básica en la prevención del consumo de alcohol y tabaco: retrato de una realidad. Rev Lat Am Enfermagem. 2005;13:784-9.

14. Gomide PIC. Efeitos das práticas educativas no desenvolvimento do comportamento antissocial. In: Marinho ML, Caballo VE, editors. Psicologia clínica e da saúde. Londrina: UEL; 2001. p. 33-53.

15. Silveira FG, Bertasso B, Magalhães LC. Tipologia Socioeconômica das famílias das grandes regiões urbanas brasileiras e seu perfil de gasto. Texto para discussão. Brasília: Instituto de Pesquisa Econômica Aplicada; 2003.

16. Malcon MC, Menezes; AM, Chatkin M. Prevalência e fatores de risco para tabagismo em adolescentes. Rev Saude Publica. 2003;37(1):1-7.

17. Eissenberg T, Balster RL. Initial tobacco use episodes in children and adolescents: current knowledge, future directions. Drug Alcohol Depend. 2000;59 Suppl $1: S 41-60$.

18. Machado Neto AS, Andrade TM, Fernandes GB, Zacharias HP, Carvalho FM, Machado AP, et al. Reliability of a questionnaire on the use of drugs by school-age adolescents, Bahia - Brazil. Rev. Saude Publica. 2010;44(5):830-9.

19. Hansen WB, Malotte CK, Fielding JE. The bogus pipeline revisited: the use of the threat of detection as a means of increasing self-reports of tobacco use. J Appl Psychol. 1985;70(4):789-92.

20. Hosmer DW, Taber S, Lemeshow S. The importance of assessing the fit of logistic regression models: a case study. Am J Public Health. 1991;81(12):1630-5.

21. Andersson B, Hibell B, Becker F, Choquet M, Kokkevi A, Fotiou A, et al. Alcohol and drug use among European 17-18 year old students: data from the ESPAD project. Stockholm: The Swedish Council for Information on Alcohol and Other Drugs (CAN), Council of Europe, Co-operation Group to Combat Drug Abuse and Illicit Trafficking in Drugs (Pompidou Group); 2007.

22. Brasil. Ministério da Saúde. Secretaria de Atenção à Saúde. Instituto Nacional do Câncer. Vigilância de tabagismo em escolares: dados de Salvador (BA). Rio de Janeiro: INCA; 2005.

23. Adalbjarnardottir S, Rafnsson FD. Perceived control in adolescent substance use: concurrent and longitudinal analyses. Psychol Addict Behav. 2001;15(1):25-32.

24. McCool JP, Cameron LD, Petrie KJ. Adolescent perceptions of smoking imagery in film. Soc Sci Med. 2001;52(10):1577-87.

25. Horta BL, Calheiros P, Pinheiro RT, Tomasi E, Amaral KC. Tabagismo em adolescentes de área urbana na região Sul do Brasil. Rev Saude Publica 2001;35(2):159-64.

26. Biglan A, Duncan TE, Ary DV, Smolkowski K. Peer and parental influences on adolescent tobacco use. J Behav Med. 1995;18(4):315-30.

27. Georgiades K, Boyle MH, Duku E, Racine Y. Tobacco use among immigrant and nonimmigrant adolescents: individual and family level influences. J Adolesc Health. 2006;38(4):443.e1-7.

28. Jackson C. Initial and experimental stages of tobacco and alcohol use during late childhood: relation to peer, parent, and personal risk factors. Addict Behav. 1997;22(5):685-98.

29. Biener L, Cullen D, Di ZX, Hammond SK. Household smoking restrictions and adolescents' exposure to environmental tobacco smoke. Prev Med. 1997;26(3):358-63.

30. Wagner MF, Oliveira MF. Habilidades sociais e abuso de drogas em adolescentes. Psic Clin. 2007;19(2):101-16.

\section{Sobre os autores}

\section{Adelmo de Souza Machado Neto}

Professor. Curso de Pós-Graduação em Medicina e Saúde, Faculdade de Medicina da Bahia, Universidade Federal da Bahia, Salvador (BA) Brasil.

\section{Tarcisio Matos Andrade}

Professor. Curso de Pós-Graduação em Medicina e Saúde, Faculdade de Medicina da Bahia, Universidade Federal da Bahia, Salvador (BA) Brasil.

\section{Carlos Napoli}

Estudante de Medicina. Escola Bahiana de Medicina e Saúde Pública, Fundação Bahiana para o Desenvolvimento das Ciências, Salvador (BA) Brasil.

\section{Lilian Carine Souza de Lima Abdon}

Estudante de Medicina. Escola Bahiana de Medicina e Saúde Pública, Fundação Bahiana para o Desenvolvimento das Ciências, Salvador (BA) Brasil.

\section{Mônica Rocha Garcia}

Estudante de Medicina. Faculdade de Tecnologia e Ciências, Salvador (BA) Brasil.

\section{Francisco Inácio Bastos}

Professor. Fundação Osvaldo Cruz - FlOCRUZ - Rio de Janeiro (RJ) Brasil. 
Anexo 1 - Variáveis independentes selecionadas para o estudo sobre o uso experimental de cigarros e o início precoce do tabagismo entre escolares na cidade de Salvador (BA), variáveis essas classificadas como sociodemográficas, relacionadas à exposição ao tabagismo de terceiros, a aspectos educacionais, à mídia e ao consumo de álcool no ano ou mês.

\begin{tabular}{|c|c|}
\hline Tipos de variáveis & Tipos de respostas \\
\hline \multicolumn{2}{|l|}{ Sociodemográficas } \\
\hline Dependência administrativa (tipo de escola) & Pública ou privada \\
\hline Gênero & Masculino ou feminino \\
\hline Curso & Médio ou fundamental \\
\hline Cor da pele (autorreferida) ${ }^{\mathrm{a}}$ & Branca/asiática ou afro-brasileira/indígena \\
\hline Estado civil dos pais & $\begin{array}{l}\text { Vivem juntos (ou casados) ou separados } \\
\text { (solteiros, divorciados, desquitados ou viúvos) }\end{array}$ \\
\hline Classe econômica $^{\mathrm{b}}$ & B1-A1 ou E-C1 \\
\hline \multicolumn{2}{|l|}{ Relacionadas à exposição ao tabagismo de terceiros } \\
\hline Familiares (pai; mãe; pai e mãe; irmãos; primos; e tios) & Sim ou não \\
\hline Pares (amigos; e namorados) & Sim ou não \\
\hline Professores & Sim ou não \\
\hline \multicolumn{2}{|c|}{ Relacionadas a aspectos educacionais, à mídia e ao consumo do álcool } \\
\hline Frequência da abordagem dos pais sobre drogas com seus filhos & Nunca/raramente ou frequentemente/sempre \\
\hline $\begin{array}{l}\text { Forma de correção dos pais em relação aos filhos quando fazem } \\
\text { algo errado }\end{array}$ & Com diálogo/limites ou agressiva/coercitiva \\
\hline Abordagem dos professores sobre drogas & Sim ou não \\
\hline $\begin{array}{l}\text { Frequência anual com que a escola faz esclarecimentos sobre o } \\
\text { tema drogas }\end{array}$ & $1-2$ vezes ou 3-6 vezes \\
\hline Proibição dos pais para o uso de cigarros pelos filhos & Sim ou não \\
\hline Influência da mídia indiretac & Sim ou não \\
\hline Consumo de álcool no ano; no mês precedente à pesquisa & Sim ou não \\
\hline
\end{tabular}

${ }^{a}$ Fonte: Fundação Instituto Brasileiro de Geografia e Estatística. ${ }^{\text {F} F o n t e: ~ A s s o c i a c ̧ a ̃ o ~ B r a s i l e i r a ~ d e ~ E m p r e s a s ~ d e ~ P e s q u i s a . ~}$ 'Filmes e espetáculos. 